\title{
An Application for the Impendance Spectroscopy Method and Building Material Testing
}

\author{
M. Luňák* \& I. Kusák \\ Department of Physics, Faculty of Civil Engineering, Brno University of Technology \\ *Corresponding author: lunak@dp.fce.vutbr.cz
}

DOI: $10.2478 / \mathrm{v} 10158-010-0009-\mathrm{x}$

\begin{abstract}
The impedance spectroscopy is used to draw information concerning electrical properties of liquids and solids. Its principles can be used easily to advantage without any prior knowledge of electrochemistry. To apply the impedance spectroscopy method at the Physics Department, Faculty of Civil Engineering, TU Brno, the method had to be adapted to the laboratory environment conditions. Furthermore, a dedicated software had to be created for this method-based diagnostics. The author of this paper has created an application called IS_alpha, which meets these requirements. Measurements employing this application are fast and convenient.
\end{abstract}

KEY WORDS: Impedance spectroscopy, solid state, measurement automation, Agilent 33220A generator, Agilent 54645A double-channel oscilloscope, IS_alpha software.

\section{INTRODUCTION}

Most testing methods being applied in civil engineering research at present are of a destructive character. These methods feature a significant drawback related to the damage the material or structure unavoidably suffers during the test: they can be applied to a particular material, unit or component only once. Non-destructive testing methods (NDTs) are free of this limitation, this being their indisputable advantage. On the other hand, their exploitation in testing laboratories, as well as in practice, still remains at a very low level. Therefore, NDT methods constitute a significant component of today's building research.

Acoustic NDT methods, such as acoustic emission (AE), Impact-Echo (IE) and ultrasonic spectroscopy (USS) make up the subject of research and development most frequently. Whereas the acoustic emission method is applicable to homogeneous materials under load, the Impact-Echo and ultrasonic spectroscopic methods apply to the materials which are free of load. All acoustic methods are based on the phenomenon of acoustic wave propagation in materials. It is to be noted that new electric testing methods, particularly the electromagnetic emission (EMG) method, have emerged recently which take advantage of the electromagnetic signal generation accompanying the formation and development of cracks in a material in the course of its time-dependent or climatic load or an external force action.

The present paper aims at contributing to the development of non-destructive testing methods in the building material testing field. It is our intention to present and investigate a new NDT method which has not yet been published in building industry periodicals, namely, the impedance spectroscopy (IS) method. The method is based on the measurement and analysis of electrical impedance characteristics of the building material 
under investigation. The essence of the mentioned method consists of analyzing the dielectric losses versus frequency plots. The method is applicable to the testing of low-electricalconductivity building materials, such as plastics, glasses, ceramics, cements, aggregates, and a number of other materials. We will show that this method is fast-operating and its results are easily reproducible. On the other hand, this method shows some applicability restrictions. It cannot be applied to thick materials $(\mathrm{d}>1 \mathrm{~cm})$ and/or to other than low-electrical-conductivity materials. Although the method has already been investigated and published to a large extent in other branches, such as biophysics and electrochemistry, its research is at its very beginning in the building industry. It is for this reason, for the time being, we would rather consider it to be a supplementary method, to be combined with other well established and better-examined NDT methods. The research of this method should no doubt be continued, with the aim of discovering more relations between the material structure and its electric response.

Measurement automation and instrumentation design and implementation, which are focused on a particular method, have proved to be a must for any experimenter since the advent of experimental physics computerization. Thanks to the instrumentation manufacturers' efforts aimed at reaching compatibility with the present-time computer operating systems, automated measurements are easier to realize. This applies not only to the impedance spectroscopy method but also to other methods using available measuring instruments. In this way, the measurements are becoming faster and less exacting for the experimenter.

The impedance spectroscopy (IS) method has been designed to investigate the electrical properties of materials featuring various electrical conductivity values. Its principles have been described elsewhere [1][2]. At the Department of Physics, Faculty of Civil Engineering, TU Brno, the IS-based measurements have been implemented using the following instrumentation: Agilent 33220A generator, Agilent 54645A double-channel oscilloscope, HP 82350 PCI HP-IB Interface card, and a PC. To operate the above-mentioned instruments and to process the IS data acquired, software called IS_alpha has been prepared by the author of this paper.

\section{EXPERIMENT SETUP DESCRIPTION}

The instruments listed above are interconnected by means of GPIB (IEEE-488) cables and connected to the Type 82350 PCI HP-IB Interface card (or any equivalent type), which is plugged into a computer running WIN XP (or higher) operating system.

Agilent 33220A generator: frequency range, $20 \mu \mathrm{Hz}$ to $20 \mathrm{MHz}$ (for sine wave output), the output waveform can be set within certain limits, the current setting being shown in the built-in LCD display. All settings can be made either using a numerical keyboard or by a rotary knob. Any setting changes take effect immediately. It can also be used to generate amplitude, frequency and impulse modulated output voltages. It can also be operated via GPIB, USB and LAN interfaces, providing compatibility with SCPI (VISA) (standard commands for program operated instruments).

Agilent 54645A double-channel oscilloscope: This is a general-purpose A/D oscilloscope featuring a bandwidth of $100 \mathrm{MHz}$, a sampling rate of $200 \mathrm{MSa} / \mathrm{s}$, an internal memory of $1 \mathrm{MB}$ for each channel. Its vertical sensitivity ranges from $1 \mathrm{mV} / \mathrm{div}$ to $5 \mathrm{~V} / \mathrm{div}$, for an input impedance of $1 \mathrm{M} \Omega(\sim 13 \mathrm{pF})$. It is operated via an IEEE-488 interface through a built-in auxiliary module, HP 54657A. Display modes available: Averaging when smoothing, the arithmetic mean is calculated from 200 successive values to be saved in the memory; this is the running average-based smoothing. In the normal mode operation, only 4000 out of one million randomly selected samples are displayed. In this case, narrow 
pulses may happen not to be displayed with a slowly-running oscilloscope time-base setting at all. For example, a 50 ns impulse could be captured and displayed by as many as 10 samples $(200 \mathrm{MSa} / \mathrm{s}=5 \mathrm{~ns} / \mathrm{sample})$. When a high-speed time base is used, the segment to be displayed consists of several short segments ("triggers"). The smoothing mode is used when the sampling rate $(200 \mathrm{MSa} / \mathrm{s})$ exceeds that of the sample saving rate in the display memory. For example, if the TB rate $>2 \mathrm{~ns} / \mathrm{d}$, several samples are merged by averaging into a single one to reach a better trace definition on the screen. Time base ranges: $2 \mathrm{~ns} / \mathrm{div}$ to $50 \mathrm{~s} /$ div. Resolution: up to $50 \mathrm{ps}$. Autoscale mode - enabled for $>10 \mathrm{mVp}-\mathrm{p}$, searching in $20 \mathrm{~ms}$ intervals.

PC-plug-in Agilent 82350 PCI HP-IB Interface card: This is a standard high-speed card, featuring an IEEE488 serial interface, a built-in buffer. It is compatible with the SICL (Standard Instrument Control Library) and VISA IO (Virtual Instrument Software Architecture) libraries for Windows 98/ME/NT/2000/XP.

\section{DESCRIPTION OF THE INSTRUMENT OPERATION AND DATA PROCESSING APPLICATION}

IS_alpha is a software which has been designed to operate measuring instruments and process IS data. The program has been written in $\mathrm{C}++$ programming language. Agilent company proprietary VISA libraries have been used when preparing the program. For the application to run smoothly, PCI-GPIB card drivers must be installed first. The application must be run under WIN XP or higher OSs. The user can run other applications without restrictions.

After the application is started, links are checked and the oscilloscope and generator are powered ON. If the above check is successful, the operator will be faced with a userfriendly environment, Fig. 1., with of course no data acquired at this stage.

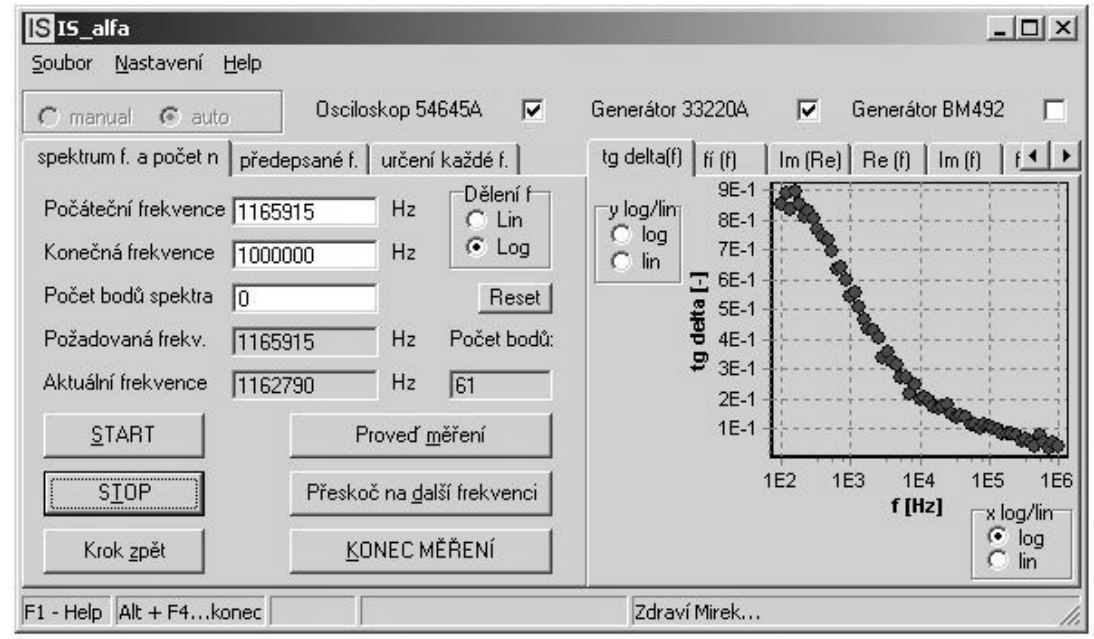

Figure 1: Alpha_IS application program interface.

The application "remembers" the settings made last time before the application was closed. The operator may therefore run their measurements immediately. Prior to quitting, they will be alerted by a sound signal. To modify the adjustable parameters, an easy-to-use "menu" can be employed whose submenu item called "File" provides an option to cancel the acquired data and initiate new measurements, save the measured and processed data, and, finally, close the application correctly. The submenu item called "Setting" can be used to change the addresses (IEEE-488) at which the system instruments are logged in. 
As a further option, the following quantities can be entered: the maximum voltage of the signal voltage source and the maximum measuring sample count, the frequency spectrum to be used for characterization, the required oscilloscope B-channel input resistance and capacitance, the Agilent generator output rms voltage, the current measurement run name (code) to be used automatically while saving the data, measurement accuracy improvement or acceleration measures to be taken, such as the measurement repetition number for each spectrum point and the measurement time separation. The last "menu" item is "Help", the meaning of which being obvious. The next line shows the Agilent instruments being used. An option for manual operation of the sine wave generator can be clicked here. In this case, the application instructs the operator in how to navigate through the measurement sequences, thus carrying out a slow semi-automatic measurement. It is apparent from Fig. 1 that the application allows the user to select the initial and end frequencies, the spectrum point count and the spectrum frequency distribution: linear or logarithmic. The operator is informed continuously of the measurement status during the measurement process. This is shown in the left-hand-side part of the IS_alpha application window. The right-hand-side provides previews of the $\operatorname{tg} \delta(\mathrm{f}), \varphi(\mathrm{f}), \operatorname{Im}(\mathrm{Re}), \operatorname{Re}(\mathrm{f}), \operatorname{Im}(\mathrm{f})$ plots, and, in the last tab, the measured data (frequency, phase, P). (Key to the diagram symbols and parameters - see [2][3][4].)

In addition to viewing the frequency data generated ( $1 \mathrm{st}$ tab in the window left-hand-side), the operator can also use default values for their measurements (2nd tab, Fig 2) or set the frequencies individually (3rd tab, Fig. 3).

Provided the measurement has been successfully completed, the operator sets the measured data save operation on target location. The application will create the following files at the selected location:

name date time year tandelta f.epw; name date time year im f.epw;

name_date_time year_im_re.epw; name_date_time_year_re_f.epw,

which can be opened immediately in the Easy Plot graphic editor. The last file having been created is name date time year.mir, in which all measured data are written in text form, to be presented in the IS alpha right-hand-side window. This file is ready to be processed by other dedicated applications, e.g., transforming selected data for use in modeling $\mathrm{S} / \mathrm{W}$ applications.

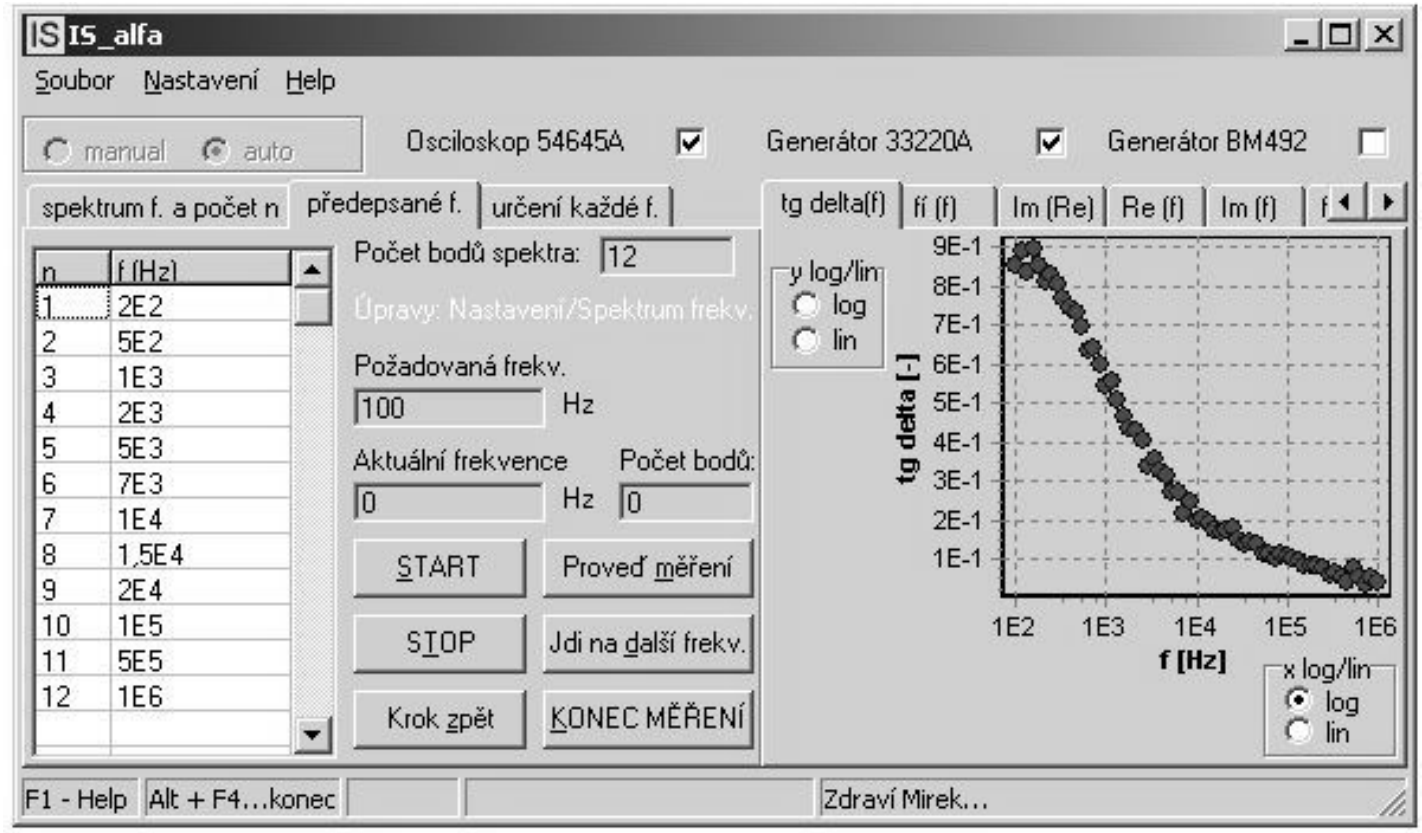

Figure2: Other options of IS_alpha-based measurements. 


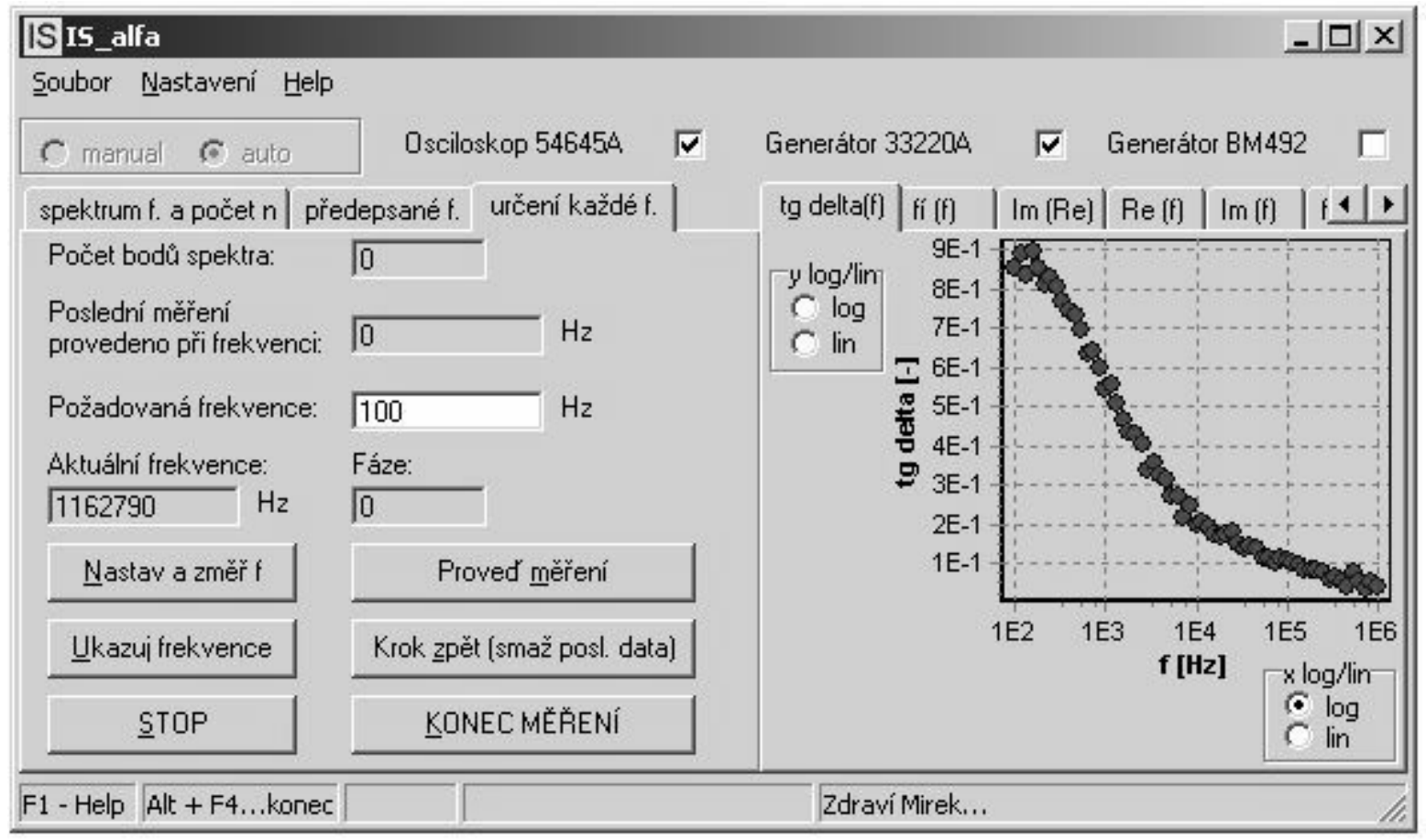

Figure3: Entering the different spectrum frequencies - the operator's panel.

\section{CONCLUSION}

The IS_alpha application meets the requirements for an IS method based automated measurement specialization. Such a specialized mode of usage can also be seen as a disadvantage. Nevertheless, its application appears to be more than adequate in the laboratory environment conditions of the Physics Department, Faculty of Civil Engineering. The measurement results achieved by means of the above application operating the IS method are presented elsewhere in these Proceedings.

\section{ACKNOLEDGEMENT}

This paper was prepared as part of a Grant Agency of the Czech Republic under Postgraduate Grant No. GACR 103/09/P263. The author offers thanks for this support herewith. The author expresses his thanks to the Department of Physics, Faculty of Civil Engineering, UT Brno, for the indispensable appliances being provided, and, last but not least, to Ass. Prof. L. Pazdera for the long-term loan of the Agilent generator.

\section{REFERENCES}

Luňák, M., Kusák, I.: Debye's Model of Impedance Spectroscopy, in: InterTech 2009 II. International Interdisciplinary Technical Conference of Young Scientist, 277-279, Politechnika Poznan, Polsko, 2009, ISBN 978-83-926896-1-4.

Kusák, I., Luňák, M., Pazdera, L.: Impedanční spektroskopie samozhutnitelného betonu při tuhnutí, in: NDT WELDING BULLETIN 2/2008, ročník 18, 22-25, vydáno 10.2008, ISSN 1213-3825. 
Luňák, M., Kusák, I.: Impedance Spectroscopy Measurement Of Concrete Hydration By Miscellaneous Medium, in: InterTech 2008 - I International Interdisciplinary Technical Conference of Young Scientist, 122-124, Politechnika Poznan, Polsko, 2008, ISBN 97883-926896-0-7.

Schauer P., 2001. Transport and Noise properties of CdTe(Cl) Crystals. Microelectronics Reliability vol. 41, iss. 3, 431-436.

Kusák, I.: Impedance spectroscopy of ceramics featuring soil and watter glass admixtures, in: New Trends in Physics 2007, 15-16.11.2007, ed. by Zdeněk Novotný Brno, 2007, 8285, ISBN 978-80-7355-078-3.

Smutny J, Pazdera L New techniques in analysis of dynamic parameters of rail fastening, INSIGHT , Vol. 46, Iss. 10, 612-615, 2004.

Luňák, M., Kusák, I., Schauer P.: Metoda Impedanční spektroskopie, in: Workshop NDT 2004, Non-Destructive Testing, 1.12.2004, ed. by Brno University of Technology, 2004, 106-108, ISBN 80-7204-371-4.

Korenska M, Pazdera L, Ritickova L. Study of correlation between acoustic emission and damaging of reinforced concrete, Conference Information: 6th International Conference of the Slovenian-Society-for-Non-Destructive-Testing, SEP 13-15, 2001 PORTOROZ, SLOVENIA, Pages: 181-184, Published: 2001. 